\title{
Improving the homologous recombination efficiency of Yarrowia lipolytica by grafting the heterogenous component from Saccharomyces cerevisiae
}

\author{
Qingchun $\mathrm{Ji}^{1}$, Jie Mai ${ }^{1}$, Ying Ding ${ }^{1}$, Yongjun $\mathrm{Wei}^{2}$, Rodrigo Ledesma-Amaro ${ }^{3}$, and \\ Xiao-Jun $\mathrm{Ji}^{1}$ \\ ${ }^{1}$ Nanjing Tech University \\ ${ }^{2}$ Zhengzhou University \\ ${ }^{3}$ Imperial College London
}

September 10, 2020

\begin{abstract}
The oleaginous non-conventional yeast Yarrowia lipolytica has enormous potential as a microbial platform for the synthesis of various bioproducts. However, while the model yeast Saccharomyces cerevisiae has very high homologous recombination (HR) efficiency, non-homologous recombination is dominant in Y. lipolytica, and foreign genes are randomly inserted into the genome. Consequently, the low HR efficiency greatly restricts the genetic engineering of this yeast. In this study, RAD52, the key component of the HR machinery in S. cerevisiae, was grafted into Y. lipolytica to improve HR efficiency. The gene ade2, whose deletion can result in a brown colony phenotype, was used as the reporter gene for evaluating the HR efficiency. The HR efficiency of Y. lipolytica strains before and after integrating the ScRad52 gene was compared using insets with homology arms of different length. The results showed that the strategy could achieve gene targeting efficiencies of up to $95 \%$ with a homology arm length of $1000 \mathrm{bp}$, which was 6.5 times of the wildtype strain and 1.6 times of the traditionally used ku70 disruption strategy. This study will facilitate the further genetic engineering of Y. lipolytica to make it a more efficient cell factory for the production of value-added compounds.
\end{abstract}

\section{Introduction}

The oleaginous non-conventional yeast Yarrowia lipolytica, a generally recognized as safe (GRAS) microorganism, is an attractive production host with great promise for industrial applications [1-4]. It has been used as a model organism to study the biosynthesis of peroxisomes, the accumulation of lipids, as well as the production and secretion of organic acids and heterologous proteins [5-7]. Due to its ability to grow using hydrophobic carbon sources, as well as generate and accumulate large amounts of lipids, it is widely used as a production host for valuable bioproducts, such as organic acids, omega-3 eicosapentaenoic acid, and other fatty acid derivatives [7-10]. In addition, it can secrete high levels of natural and heterologous proteins and has been used to produce various heterologous proteins, including proteases, lipases, and RNases [6, 11].

Targeted deletion or insertion of genes and functional nucleic acid sequences at the target sites of a microbial genome is not only an effective method to study the gene function and regulate gene expression, but also a basic tool for genome editing for strain improvement. With the deciphering of the whole-genome sequence of $Y$. lipolytica [12], a series of platforms for molecular biology and genetics were established in this yeast, including transformation methods for gene deletion and integration [13, 14], which made it possible to redesign whole metabolic pathways [15]. However, in $Y$. lipolytica, the vast majority of foreign genes are randomly inserted into the genome, and the probability of targeted insertion by homologous recombination 
(HR) is extremely low [16-18]. When the 5'- and 3'-flanking regions of the target gene are 0.5-1.0 kb long, HR occurs only at a rate of $0-36 \%$ [19]. This is due to the fact that $Y$. lipolytica has a dominant non-homologous end joining (NHEJ) recombination pathway that it uses to repair the DNA double strand breaks (DSB) rather than the HR pathway [20]. These two repair mechanisms are independent and competitive [21]. Most eukaryotic microorganisms use NHEJ as the main DSB repair pathway, and knocking out or destroying NHEJ-related genes is an effective strategy to increase the efficiency of gene targeting. However, many studies also showed that disrupting the NHEJ pathway can make the cells prone to mutation, which is not conducive to industrial application [22, 23]. Moreover, the cell growth can be severely affected under non-optimal conditions, such as high temperature, ultraviolet irradiation and the presence of chemical DNAdamaging agents $[24,25]$. In addition, in strains lacking components of the NHEJ pathway, the integration efficiency is highly dependent on the targeted gene locus.

Consequently, strengthening the HR repair is also an effective approach for improving the gene targeting efficiency. The HR repair pathway is mediated by a class of conserved enzymes called DNA recombinase enzymes, most of which were initially identified in Saccharomyces cerevisiae [26]. Notably, the RAD51/RAD52 complex is a major participant in the targeted integration of foreign DNA in eukaryotes and plays a key role in the HR pathway [27] (Fig. 1). S. cerevisiae has very high HR efficiency (up to 100\%), and ScRAD52 has been identified as an important component that plays a vital role in mitotic and meiotic recombination in this organism [28-30]. The heterologous expression of S. cerevisiae HR related genes such asScRad51 and ScRad52, in mammalian cells (e.g., monkey embryonic cells) and plant cells (e.g., Arabidopsis ), or its combination with the CRISPR/Cas9 system, can greatly increase the efficiency of HR and reduce the off-target effects [31-36].

Here, we focused on improving the HR efficiency of $Y$. lipolyticaby grafting heterologous component that is crucial for the HR mechanisms of $S$. cerevisiae. Since ScRad52 is a key repair gene mainly responsible for DSB repair, we hypothesized that heterologous expression of the $S c$ RAD52 protein in $Y$. lipolytica would improve its HR efficiency. To validate this concept, the ScRad52 gene was first heterologously expressed in $Y$. lipolytica and the HR efficiency was examined by knocking out the adenosine succinic acid synthase gene (ade2, YALI0B23188g) using cassettes with homology arms of different lengths. The corresponding endogenous gene of Y. lipolytica (YlRad52, YALI0F02431g) was further knocked out to explore its role in $Y$. lipolytica and whether the heterologous expression of ScRAD52 could replace the function of the native YlRAD52 protein. This work provides an effective strategy for precise genome editing, which may contribute to the molecular biology and genetics of $Y$. lipolytica and will greatly facilitate the rapid development of genome editing in other similar microorganisms.

\section{Materials and methods}

\subsection{Strains and culture conditions}

Y. lipolytica Po1f (ATCC MYA-2613), a leucine and uracil auxotrophic strain, was used as the base strain for all genome editing and expression experiments in this study. The ku70-disrupted $Y$. lipolytica strain Po1f- $\Delta$ ku70 was constructed using the URA3-blaster method and a ku70 deletion cassette as described by Jang et al. [16]. Escherichia coli DH5 $\alpha$ was used for plasmid construction and propagation. It was grown in Luria-Bertani medium supplemented with $100 \mathrm{mg} / \mathrm{L}$ ampicillin at $37^{\circ} \mathrm{C}$. Yeast cultures were grown in YPD medium $\left(20 \mathrm{~g} / \mathrm{L}\right.$ glucose, $10 \mathrm{~g} / \mathrm{L}$ yeast extract, and $20 \mathrm{~g} / \mathrm{L}$ tryptone) at $28^{\circ} \mathrm{C}$. The synthetic complete medium without uracil (SC-Ura) plates contained $20 \mathrm{~g} / \mathrm{L}$ glucose, $6.7 \mathrm{~g} / \mathrm{L}$ yeast nitrogen base (YNB) without amino acids (BBI, Sangon Biotech (Shanghai) Co. Ltd., China), $0.77 \mathrm{~g} / \mathrm{L}$ CSM-Ura (Complete supplement mixture minus uracil, MP Biomedicals, USA), and $20 \mathrm{~g} / \mathrm{L}$ agar. The synthetic complete medium without leucine (SCLeu) plates contained $20 \mathrm{~g} / \mathrm{L}$ glucose, $6.7 \mathrm{~g} / \mathrm{L}$ YNB without amino acids (BBI, Sangon Biotech (Shanghai) Co. Ltd., China), $0.77 \mathrm{~g} / \mathrm{L}$ CSM-Leu (Complete Supplement Mixture minus leucine, MP Biomedicals, USA), and $20 \mathrm{~g} / \mathrm{L}$ agar. The URA3 maker was removed and selected against on SC+FOA plates, which contained $6.7 \mathrm{~g} / \mathrm{L}$ YNB, $0.77 \mathrm{~g} / \mathrm{L}$ CSM (Complete Supplement Mixture, MP Biomedicals, USA), $0.8 \mathrm{~g} / \mathrm{L}$ 5-fluoroorotic acid, and $20 \mathrm{~g} / \mathrm{L}$ agar. 


\subsection{General molecular biology techniques}

The plasmids and primers used in this study are listed in Table 1 and Supplementary Table 1. Genomic DNA from the yeast strains was purified using an E.Z.N.A. ${ }^{\circledR}$ Yeast DNA Kit (Omega Bio-Tek Inc., USA). The transformation of Y. lipolytica was performed using the Frozen-EZ Yeast Transformation II Kit (Zymo Research, Orange, CA). Gene deletion was performed by HR based on direct repeats, using URA3 as a counter-selection marker on SC+FOA plates. The recombinant strains were spread on SC+FOA plates and cultured for 2-3 days. Strains expressing URA3 did not grow in the presence of 5-FOA.

\subsection{Construction of expression cassettes expressing ScRad52}

The S. cerevisiae Rad52 sequence (GenBank accession No. CAA86623.1) was codon-optimized for expression in Y. lipolyticaand ordered as fully synthetic DNA (GeneScript, Nanjing, China, Supplementary Table 2). The vector pUC-Leu-A08-ScRad52 expressing the gene of interest under the control of the endogenous Rad52 gene promoter $\mathrm{P}_{\mathrm{YlRad} 52}$ and terminator $\mathrm{T}_{\text {YlRad52 }}$ as generated using the ClonExpress ${ }^{\mathrm{TM}}$ MultiS one step cloning kit (Vazyme, Nanjing, China), according to the manufacturer's instructions. The promoter PYlRad52 and terminator TYlRad52 were respectively amplified by PCR using the primer pairs PYlRad52-F/R and TYlRad52-F/R with genomic DNA extracted from Y. lipolytica. The codon-optimized ScRad52 gene was then amplified using the primers ScRad52-F/R. The PCR-amplified sequences were digested with Pac I and Sna BI, and then one-step cloned into the digested pUC-Leu-A08 backbone to generate the recombinant vector pUC-Leu-A08-ScRad52. We used the A08 gene site in the $Y$. lipolytica genome whose disruption is known to not affect cell growth [37]. All the newly constructed plasmids were confirmed by DNA sequencing.

\subsection{Construction of disruption cassettes}

Disruption cassettes targeting YlRad52 (YALI0F02431g) andade2 (YALI0B23188g) were constructed according to the URA3-blaster protocol [16]. For the construction of the YlRad52 deletion cassette, $2 \mathrm{~kb} 5$ 'and 3'- homology arms of the YlRad52 gene, named YlRad52-up and YlRad52-down, were respectively amplified from Y. lipolytica genomic DNA using the primer pairs YlRad52-up-F/R and YlRad52-down-F/R. The resulting PCR fragments were respectively digested with Eco RI and Pac I, and one-step cloned into the pUC-HUH vector containing the HisG-URA3-HisG (HUH) blaster cassette, to generate the vector pUC-HUH- ${ }^{Y} l R a d 52$.

For the construction of the ade2 deletion cassettes with 5'- and 3'-homology arms of 1000, 500, 250 and $100 \mathrm{bp}$, the homology fragments were respectively amplified from the yeast genomic DNA using the primer combinations 1000-up-F, 500-up-F, 250-up-F, 100-up-F/Ade2-up-R and Ade2-down-F/1000-down-R, 500down-R, 250-down-R, 100-down-R. The obtained PCR fragments were respectively digested with Eco R I andPac I, and one-step cloned into the pUC-HUH vector containing the HisG-URA3-HisG blaster cassette, to generate the vectors of pUC-HUH- $\Delta$ ade2-1000, pUC-HUH- $\Delta$ ade2-500, pUC-HUH- $\Delta$ ade2-250, and pUCHUH- $\Delta$ ade2-100.

\subsection{Determination of the homologous recombination efficiency}

The ade2 gene was selected as the reporter, because its deletion causes the accumulation of phosphoribosyl amino imidazole, resulting in the disrupted mutants becoming brown (Supplementary Fig. 1). The HR efficiency was estimated by counting the number of de2 $^{-}$- colonies arising from cells transformed with the ade2 disruption cassettes with different homology arm lengths (100, 250, 500 and 1,000 bp) [49]. The HR efficiency at the ade 2 locus was determined via the ratio of brown colonies. Transformation with the disruption cassettes was repeated three times.

\section{Results}

\subsection{Construction of recombination strains expressing ScRad52}

The expression cassette encoding the ScRad52 gene driven by the endogenous promoter PYRad52 and URA3blaster expression cassette were integrated into the A08 locus in the genomes of the $Y$. lipolytica strains Po1f and Po1f- $\Delta$ ku70. The resulting strains $Y$. lipolytica Po1f-ScRad52 and Po1f- $\Delta$ ku70-ScRad52 were successfully 
selected on SC-Ura plates, and further confirmed by colony PCR as well as genomic DNA isolation followed by PCR. Microscopic examination and colony morphology observation throughout the growth cycle indicated that the engineered $Y$. lipolytica was not morphologically different from the parental strain.

\subsection{ScRad52 expression enhanced the homologous recombination efficiency}

To explore the effect of heterologous expression of the ScRad52gene on the HR efficiency of $Y$. lipolytica , we selected genes whose deletion results in significant phenotypic changes (e.g. color, fluorescence, etc.) as target sites. In yeast, the adenylosuccinate synthetase gene ade2 is responsible for the conversion of phosphoribosyl aminoimidazole (AIR) into 5-amino 4-carboxyimidazoleribose (CAIR). The deletion of ade2 causes the accumulation of AIR, which turns the corresponding yeast colonies brown (Fig. 2). This phenotype was used for easy visual selection in bothS. cerevisiae and Y. lipolytica [38-40]. Therefore, the ade2 was chosen as the target site for gene knockout in this study.

By using the URA3 blaster method with homology arms of different targeting the ade2 gene as a single variable, knockout plasmids containing different homology arm lengths were constructed via a single-fragment one-step assembly method. After the recombinant plasmid was linearized and introduced into $Y$. lipolytica , the positive transformants were screened according to their visible phenotype and confirmed using colony PCR. The HR efficiency of $Y$. lipolyticastrains, including Po1f-ScRad52 and Po1f- $\Delta$ ku70-ScRad52, as well as the parental strains Polf and Po1f- $\Delta$ ku70, was obtained by calculating the ratio of the colonies with ade2 knockout to the total number of strains on the plate, that is, the ratio of brown mutants.

As shown in Table 2, when the ade2 knockout was performed with homology arms of $1000 \mathrm{bp}$, HR efficiency of the engineered strains $Y$. lipolytica Po1f-ScRad52 and Po1f- $\Delta$ ku70-ScRad52 with heterologous HR components from S. cerevisiae reached $90 \%$ and $95 \%$, respectively. By contrast, the HR efficiency of the control strains Y. lipolytica Po1f and Po1f- $\Delta$ ku70 was only $14.7 \%$ and $60 \%$, respectively. The best strain with the highest HR efficiency for ade2 deletion was $Y$. lipolytica Po1f- $\Delta$ ku70-ScRad52. Its HR efficiency reached up to $95 \%$, which was 6.5 times of the wild-type strain Y. lipolytica Po1f (Table 3). These results indicated that ScRAD52, the main component of the HR machinery in S. cerevisiae, was functional and improved the HR efficiency in $Y$. lipolytica, simplifying genome editing.

\subsection{Effect of homology arm length on homologous recombination efficiency}

Disruption cassettes with homology arms of 1000, 500, 250, 100 and 50 bp targeting the ade2 were constructed. As shown in Table 2, when the disruption cassette with 1000 bp homology regions was used for ade2 deletion, the percentage of transformants generated by HR in the strains Po1f-ScRad52 and Po1f- $\Delta$ ku70ScRad52 reached $60 \%$ and $95 \%$, respectively. However, when the length of homology arms was less than $500 \mathrm{bp}$, the HR efficiency dropped sharply. At $100 \mathrm{bp}$, the HR efficiency of Y. lipolytica Po1f-ScRad52 and Po1f- $\Delta$ ku70-ScRad52 decreased to $6.1 \%$ and $11.9 \%$ respectively, which was only $6.8 \%$ and $12.5 \%$ of that with homology arms of $1000 \mathrm{bp}$ (Fig. 3). The extension of homology arm length on each side of the disruption cassette increased the HR efficiency, which was consistent with previous reports [40-42].

\subsection{Effect of endogenous Rad52 disruption on homologous recombination efficiency}

In order to further investigate the effect of the endogenous Ras52 (YlRad52) on the HR efficiency, and whether the heterologousScRad52 can completely replace YlRad52 in Y. lipolytica, the YlRad52 gene was knocked out using the URA3 blaster method in the aforementioned $Y$. lipolytica strains. The growth rate of the engineered strains with the YlRad52 gene knockout was slightly lower than that of the YlRad52 wild-type strains. In addition, for the two strains Po1f-ScRad52- $\Delta$ YlRad52 and Po1f- $\Delta$ ku70-ScRad52$\Delta$ YlRad52, there was almost no difference of colony morphology compared to the parental strains. However, the strains Po1f- $\Delta$ YlRad52 and Po1f- $\Delta$ ku70- $\Delta$ YlRad52 formed smaller and sharper colonies on YPD plates compared with the other two strains Po1f-ScRad52-YlRad52 and Po1f- $\Delta$ ku70-ScRad52- $\Delta$ YlRad52 which express theScRad52 gene. This may be explained by the fact that Rad52is important for nuclear integrity in Y. lipolytica. This result is similar to a report by Campos-Gongora et al. [26]. The expression of the ScRad52 gene alleviates the damage to nuclear integrity caused by the deletion of the endogenous 
Y. lipolyticaRad52 to some extent. Consequently, the colony morphology of Po1f-ScRad52- $\Delta$ YlRad52 and Po1f- $\Delta$ ku70-ScRad52- $\Delta$ YlRad52 was not significantly different from the original strains.

The HR efficiency of the strains Po1f-ScRad52- $\Delta$ YlRad52 and Po1f- $\Delta$ ku70-ScRad52- $\Delta$ YlRad52 was significantly lower than the HR efficiency of the strains with their own YlRad52 genes (Table 2 and Fig. 3). When the homology arm length was $1000 \mathrm{bp}$, the HR efficiency of strain Po1f-ScRad52- $\Delta$ YlRad52 reached $53.3 \%$, which was only $40.8 \%$ that of Po1f-ScRad52. Moreover, the HR efficiency of Po1f- $\Delta$ ku70-ScRad52$\Delta$ YlRad52 was $6.7 \%$, which was even lower than that of the wild-type strain $Y$. lipolytica Po1f. Compared with Po1f- $\Delta$ ku70-ScRad52, the HR efficiency of Po1f- $\Delta$ ku70-ScRad52- $\Delta$ YlRad52 was reduced by $92.9 \%$, and there was practically no recombination with homology arms of 250 and $100 \mathrm{bp}$. These results show that the YlRad52 gene plays a crucial role in the native HR mechanism of $Y$. lipolytica, which is consistent with the previous report that the Rad52 gene in is crucial for DNA repair in this yeast [26].

\section{Discussion}

With the development of artificial nucleases in recent years, the possibilities of performing accurate genome editing based on HR have been greatly expanded. Although the efficiency is still relatively low, doublestrand breaks can be introduced at practically any target sits, but there are great differences in knockout efficiency for different organisms [43, 44]. RAD52 protein is considered to be the crucial recombinase of the HR pathway. Previous studies have reported that integrating the $S$. cerevisiae RAD52 can significantly enhance the efficiency of HR in mammalian cells [36]. In the present study, the gene encoding RAD52 from S. cerevisiae was codon-optimized and expressed in $Y$. lipolytica. The results showed that the heterologous expression of ScRad52 significantly improved the HR efficiency of Y. lipolytica, and the endogenous Rad52 gene also plays a crucial role in the $Y$. lipolytica HR mechanism. When the homology arm length was 1000 bp, the HR efficiency of the strains, Po1f, Po1f- $\Delta$ ku70, Po1f-ScRad52, and Po1f- $\Delta$ ku70-ScRad52 was 14.7\%, $60 \%, 90 \%$, and $95 \%$, respectively. The highest HR efficiency was observed in the strain Po1f- $\Delta$ ku70-ScRad52, and it was 6.5 times of the wildtype strain Polf as well as 1.6 times of the traditionally used ku70disruption strategy. However, when the he endogenous RAD52 gene (YlRad52) of Y. lipolytica was knocked out, the HR efficiency was reduced. Although the mechanism of HR mediated by YlRad52 is currently unclear, the results show that it plays a very important role in nuclear integrity and DSB repair. Interestingly, the HR efficiency of the Po1f- $\Delta$ ku70-ScRad52- $\Delta$ YlRad52 strain was lower than that of Polf-ScRad52- $\Delta$ YlRad52, which may be due to the deletion of the KU70 protein related to the NHEJ mechanism in this strain, further compromising nuclear integrity and DNA repair in this strain compared to Po1f-ScRad52- $\Delta$ YlRad52. In addition, in the engineered strain with the YlRad52 knockout, the increase of homology arm length improved the HR efficiency. Therefore, HR mediated by YIRAD52 plays a very important role in nuclear integrity and DNA repair.

In conclusion, the HR efficiency of $Y$. lipolytica was improved by integrating the key gene ScRad52 governing HR in S. cerevisiae. The HR efficiency of the best engineered strain Po1f- $\Delta$ ku70-ScRad52 reached $95 \%$ with $1000 \mathrm{bp}$ homology arms. Additionally, the results proved that the endogenous YIRAD52 plays an important role in HR. Generally, most industrially important non-model eukaryotic microorganisms have a complicated genetic background and are inherently inefficient in terms of HR, making it difficult to achieve large-scale, high-throughput gene editing in these cells. This study provides a reference for improving the HR efficiency of $Y$. lipolytica and other similar eukaryotic microorganisms, which can further accelerate the genome editing and metabolic engineering of important industrial hosts.

\section{Acknowledgement}

This work was financially supported by the National Science Fund for Excellent Young Scholars of China (No. 21922806), the National Key Research and Development Program of China (No. 2018YFA0903700), the National Natural Science Foundation of China (Nos. 21776131, and 22061130203), the Six Talent Peaks Project in Jiangsu Province of China (No. 2018-SWYY-047), the Jiangsu Synergetic Innovation Center for Advanced Bio-Manufacture (No. XTD1814), Key Research and Development Program of Jiangsu Province, and the BBSRC grant BB/R01602X/1. 


\section{Conflict of interest}

The authors declare no financial or commercial conflict of interest.

\section{References}

1. Liu, H. H., Ji, X. J., Huang, H., Biotechnological applications of Yarrowia lipolytica : Past, present and future. Biotechnol. Adv. 2015, 33, 1522-1546.

2. Ma, Y. R., Wang, K. F., Wang, W. J., Ding, Y., et al., Advances in the metabolic engineering of Yarrowia lipolytica for the production of terpenoids. Bioresour. Technol. 2019, 281, 449-456.

3. Muhammad, A., Feng, X., Rasool, A., Sun, W., Li, C., Production of plant natural products through engineered Yarrowialipolytica . Biotechnol . Adv . 2020,43,107555.

4. Ma, J., Gu, Y., Marsafari, M., Xu, P., Synthetic biology, systems biology, and metabolic engineering of Yarrowia lipolyticatoward a sustainable biorefinery platform. J. Ind.Microbiol . Biotechnol . 2020, Doi: $10.1007 / \mathrm{s} 10295-020-02290-8$

5. Wang, J., Ledesma-Amaro, R., Wei, Y., Ji, B., Ji, X. J., Metabolic engineering for increased lipid accumulation in Yarrowialipolytica - A Review. Bioresour. Technol. 2020, 313: 123707.

6. Dulermo, R., Brunel, F., Dulermo, T., Ledesma-Amaro, R., et al., Using a vector pool containing variable-strength promoters to optimize protein production in Yarrowia lipolytica .Microb . Cell Fact . 2017, 16, 31.

7. Yu, Z., Du, G., Zhou, J., Chen, J., Enhanced $\alpha$-ketoglutaric acid production in Yarrowia lipolytica WSH-Z06 by an improved integrated fed-batch strategy. Bioresour. Technol. 2012, 114, 597-602.

8. Xue, Z., Sharpe, P. L., Hong, S. P., Yadav, N. S., et al., Production of omega-3 eicosapentaenoic acid by metabolic engineering of Yarrowia lipolytica . Nat . Biotechnol . 2013, 31, 734-740.

9. Ledesma-Amaro, R., Nicaud, J. M. Yarrowia lipolytica as a biotechnological chassis to produce usual and unusual fatty acids.Prog . Lipid Res . 2016, 61, 40-50.

10. Xu, P., Qiao, K., Ahn, W. S., Stephanopoulos, G., Engineering Yarrowia lipolytica as a platform for synthesis of drop-in transportation fuels and oleochemicals. Proc. Natl. Acad. Sci. USA . 2016, 113, 10848-10853.

11. Madzak, C., Gaillardin, C., Beckerich, J. M., Heterologous protein expression and secretion in the non-conventional yeast Yarrowialipolytica : a review. J. Biotechnol. 2004, 109, 63-81.

12. Dujon, B., Sherman, D., Fischer, G., Durrens, P., et al. Genome evolution in yeasts. Nature . 2004, $430,35-44$.

13. Larroude, M., Rossignol, T., Nicaud J. M., Ledesma-Amaro, R. Synthetic biology tools for engineering Yarrowia lipolytica .Biotechnol Adv . 2018, 36, 2150-2164.

14. Shi, T. Q., Huang, H., Kerkhoven, E. J., Ji, X. J. Advancing metabolic engineering of Yarrowia lipolytica using the CRISPR/Cas system. Appl. Microbiol . Biotechnol . 2018, 102, 9541-9548.

15. Abdel-Mawgoud, A. M., Markham, K. A., Palmer, C. M., Liu, N., et al., Metabolic engineering in the host Yarrow $i$ a lipolytica .Metab. Eng . 2018, 50, 192-208.

16. Jang, I. S., Yu, B. J., Jang, J. Y., Jegal, J., Lee, J. Y., Improving the efficiency of homologous recombination by chemical and biological approaches in Yarrowia lipolytica . PLoSOne . 2018, 13, e0194954.

17. Ding, Y., Wang, K. F., Wang, W. J., Ma, Y. R., et al., Increasing the homologous recombination efficiency of eukaryotic microorganisms for enhanced genome engineering. Appl . Microbiol .Biotechnol . 2019, 103, 4313-4324. 
18. Tsakraklides, V., Brevnova, E., Stephanopoulos, G., Shaw, A. J. Improved gene targeting through cell cycle synchronization.PLoS One . 2015, 10, e0133434.

19. Schwartz, C., Frogue, K., Ramesh, A., Misa, J., Wheeldon, I., CRISPRi repression of nonhomologous end-joining for enhanced genome engineering via homologous recombination in Yarrowialipolytica. Biotechnol . Bioeng . 2017, 114, 2896-2906.

20. Richard, G. F., Kerrest, A., Lafontaine, I., Dujon, B., Comparative genomics of hemiascomycete yeasts: genes involved in DNA replication, repair, and recombination. Mol . Biol . Evol . 2005, 22, 1011-1023.

21. van Dyck, E., Stasiak, A. Z., Stasiak, A., West, S. C., Binding of double-strand breaks in DNA by human Rad52 protein. Nature. 1999, 398, 728-731.

22. Barnes, G., Rio, D., DNA double-strand-break sensitivity, DNA replication, and cell cycle arrest phenotypes of Ku-deficientSaccharomyces cerevisiae . Proc . Natl .Acad . Sci . USA . 1997, 94, 867-872.

23. Kretzschmar, A., Otto, C., Holz, M., Werner, S., et al., Increased homologous integration frequency in Yarrowia lipolyticastrains defective in non-homologous end-joining. Curr .Gen . 2013, 59, 63-72.

24. Zhang, J., Mao, Z., Xue, W., Li, Y., et al., Ku80 gene is related to non-homologous end-joining and genome stability in Aspergillusniger . Curr . Microbiol . 2011, 62, 1342-1346.

25. Gandía, M., Xu, S., Font, C., Marcos, J. F., Disruption of ku70 involved in non-homologous end-joining facilitates homologous recombination but increases temperature sensitivity in the phytopathogenic fungus Penicillium digitatum .Fungal Biol . 2016, 120, 317-323.

26. Campos-Góngora, E., Andaluz, E., Bellido, A., Ruiz-Herrera, J., Larriba, G., The RAD52 ortholog of Yarrowia lipolyticais essential for nuclear integrity and DNA repair. FEMSYeast Res . 2013, 13, 441-452.

27. Di Primio, C., Galli, A., Cervelli, T., Zoppe, M., Rainaldi, G., Potentiation of gene targeting in human cells by expression ofSaccharomyces cerevisiae Rad52. NucleicAcids Res . 2005, 33, 4639-4648.

28. Resnick, M. A., Genetic control of radiation sensitivity inSaccharomyces cerevisiae . Genetics . 1969, 62,519 .

29. Mortensen, U. H., Erdeniz, N., Feng, Q., Rothstein, R., A molecular genetic dissection of the evolutionarily conserved N terminus of yeast Rad52. Genetics . 2002, 161, 549-562.

30. McIlwraith, M. J., West, S. C., DNA repair synthesis facilitates RAD52-mediated second-end capture during DSB repair. Mol .Cell 2008, 29, 510-516.

31. Park, M. S., Expression of human RAD52 confers resistance to ionizing radiation in mammalian cells. J. Biol . Chem .1995, 270, 15467-15470.

32. Vispé, S., Cazaux, C., Lesca, C., Defais, M., Overexpression of Rad51 protein stimulates homologous recombination and increases resistance of mammalian cells to ionizing radiation. Nucleic AcidsRes . 1998, 26, 2859-2864.

33. Yanez, R., Porter, A., Gene targeting is enhanced in human cells overexpressing hRAD51. Gene Ther . 1999, 6, 1282.

34. Shcherbakova, O. G., Lanzov, V. A., Ogawa, H., Filatov, M. V., Overexpression of bacterial RecA protein stimulates homologous recombination in somatic mammalian cells. Mutat . Res . 2000, 459, 65-71.

35. Shaked, H., Melamed-Bessudo, C., Levy, A. A., High-frequency gene targeting in Arabidopsis plants expressing the yeast RAD54 gene. Proc . Natl . Acad . Sci . USA . 2005, 102, 12265-12269. 
36. Shao, S., Ren, C., Liu, Z., Bai, Y., et al., Enhancing CRISPR/Cas9-mediated homology-directed repair in mammalian cells by expressing Saccharomyces cerevisiae Rad52. Int. J. Biochem. Cell Biol . 2017, 92, 43-52.

37. Schwartz, C., Shabbir-Hussain, M., Frogue, K., Blenner, M., Wheeldon, I., Standardized markerless gene integration for pathway engineering in Yarrowia lipolytica . ACS Synth .Biol . 2017, 6, 402-409.

38. Wagner, J. M., Williams, E. V., Alper, H. S., Developing a piggyBac transposon system and compatible selection markers for insertional mutagenesis and genome engineering in Yarrowialipolytica . Biotechnol. J. 2018, 13, e1800022.

39. Ugolini, S., Bruschi, C. V., The red/white colony color assay in the yeast Saccharomyces cerevi siae: epistatic growth advantage of white ade8-18, ade2 cells over red ade2 cells. Curr. Ge n. 1996, 30, 485-492.

40. Verbeke, J., Beopoulos, A., Nicaud, J. M., Efficient homologous recombination with short length flanking fragments in Ku70 deficient Yarrowia lipolytica strains. Biotechnol .Lett . 2013, 35, 571-576.

41. Choo, J. H., Han, C., Kim, J. Y., Kang, H. A., Deletion of a KU80 homolog enhances homologous recombination in the thermotolerant yeastKluyveromyces marxianus . Biotechnol . Lett . 2014, 36, 2059-2067.

42. Oguro, Y., Yamazaki, H., Ara, S., Shida, Y., et al., Efficient gene targeting in non-homologous endjoining-deficient Lipomycesstarkeyi strains. Curr . Gen . 2017, 63, 751-763.

43. Ran, F. A., Hsu, P. D., Lin, C.-Y., Gootenberg, J. S., et al., Double nicking by RNA-guided CRISPR/Cas9 for enhanced genome editing specificity. Cell 2013, 154, 1380-1389.

44. Rong, Z., Zhu, S., Xu, Y., Fu, X., Homologous recombination in human embryonic stem cells using CRISPR/Cas9 nickase and a long DNA donor template. Protein \& Cell 2014, 5, 258-260.

45.

Table 1. Strains and plasmids used in this study

\begin{tabular}{|c|c|}
\hline Strains or plasmids & Genotypes or properties \\
\hline \multicolumn{2}{|l|}{ Strains } \\
\hline E. $\operatorname{coli} \mathrm{DH} 5 \alpha$ & supE44 [?]lacU169 ( $\varphi 80$ lacZ [?]M15) hsdR17 recA1 endA1 gyrA96 thi-1 rel \\
\hline Y. lipolytica Po1f (ATCC MYA-2613) & MATa, leu2-270, ura3-302, xpr2-322, axp1-2 \\
\hline$Y$. lipolytica Polf- $\Delta \mathrm{ku} 70$ & Po1f, $\Delta \kappa v 70$ \\
\hline Y. lipolytica Po1f-ScRad52 & Po1f, ScRad52 ::A08 \\
\hline Y. lipolytica Po1f- $\Delta \mathrm{ku} 70-\mathrm{ScRad} 52$ & Po1f, $\Delta \kappa v 70, \Sigma_{\varsigma} P a \delta 52:: A 08$ \\
\hline Y. lipolytica Polf- $\Delta$ YlRad52 & $\Pi о 1 \varphi, \Delta \Psi \lambda P a \delta 52$ \\
\hline$Y$. lipolytica Po1f- $\Delta$ ku70- $\Delta$ YlRad52 & Po1f, $\Delta \kappa \cup 70, \Delta \Psi \lambda P a \delta 52$ \\
\hline Y. lipolytica Po1f-ScRad52 - $\Delta$ YlRad52 & Po1f, $\Sigma_{S} P a \delta 52:: A 08, \Delta \Psi \lambda P a \delta 52$ \\
\hline Y. lipolytica Po1f- $\Delta$ ku70-ScRad52-YlRad52 & Po1f, $\Delta \kappa v 70, \Sigma_{S} P a \delta 52:: A 08, \Delta \Psi \lambda P a \delta 52$ \\
\hline \multicolumn{2}{|l|}{$\begin{array}{l}\text { Plasmids } \\
\text { Plonter }\end{array}$} \\
\hline pUC57 & ColE1 Ori, LacZ, Amp \\
\hline pUC-Leu-A08 & A08 upstream and downstream homology arms and Leu auxotrophic selecti \\
\hline pUC-Leu-A08-ScRad52 & $\mathrm{P}_{\text {YlRad52-ScRad52 -T }}$ YlRad52 \\
\hline pUC-HUH & HisG-URA3-HisG (HUH) in pUC57 \\
\hline pUC-HUH- $\Delta$ YlRad52 & YlRad52-up-HUH-YlRad52-down \\
\hline pUC-HUH- $\Delta$ ade2-100 & ade2-100-up-HUH-ade2-100-down \\
\hline pUC-HUH- $\Delta$ ade2-250 & ade2-250-up-HUH-ade2-250-down \\
\hline pUC-HUH- $\Delta$ ade2-500 & ade2-500-up-HUH-ade2-500-down \\
\hline pUC-HUH- $\Delta$ ade2-1000 & ade2-1000-up-HUH-ade2-1000-down \\
\hline
\end{tabular}


Table 2. Homologous recombination efficiency of different $Y$. lipolytica strains using different homology arm lengths.

\begin{tabular}{|c|c|c|}
\hline Y. lipolytica strains & HR efficiency with different homology arm length (\%) & HR efficiency with \\
\hline & $100 \mathrm{bp}$ & $250 \mathrm{bp}$ \\
\hline $\operatorname{Po1f}^{k} u 70-S c R a d 52-{ }^{Y} l R a d 52$ & 0 & 0 \\
\hline Po1f & 0 & 2 \\
\hline Polf-ScRad52- ${ }^{Y} l \operatorname{Rad} 52$ & 1.79 & 11.9 \\
\hline $\mathrm{Po}_{\mathrm{f}-}{ }^{k} u 70$ & 3.3 & 13.3 \\
\hline Po1f-ScRad52 & 6.1 & 22.6 \\
\hline Po1f- ${ }^{k} u 70-S c R a d 52$ & 11.9 & 32.8 \\
\hline
\end{tabular}

\section{Figure legends}

Figure 1. Pathways of DNA double-strand break (DSB) repair by homologous recombination (HR) and non-homologous end joining (NHEJ) in Y. lipolytica . Y. lipolytica is more inclined to use the NHEJ pathway for DSB repair rather than the HR pathway. Destroying NHEJ-related proteins (KU70/KU80) can increase the efficiency of gene targeting. The RAD51/RAD52 complex plays a key role in the HR pathway.

Figure 2. Screening method based on deleting the adenylosuccinate synthetase gene (ade2) involved in adenine biosynthesis in $Y$. lipolytica. The deletion of the ade2 gene results in a brown colony phenotype. PRPP: phosphoribosyl pyrophosphate; PRA: 5-phosphoribosylamine, GAR: glycinamide ribotide, FGAR: formyl glycinamide ribotide, FGAM: formyl glycinamide ribotide, AIR: phosphoribosyl aminoimidazole, CAIR: 5-amino 4-carboxyimidazole ribotide.

Figure 3. Homologous recombination efficiency of wild-type $Y$. lipolytica Polf and different engineered strains. Disruption cassettes with different homology arm lengths targeting the adenylosuccinate synthetase encoding gene (ade2) were constructed. The length of the homology arms from left to right is $100,250,500$, and $1000 \mathrm{bp}$. The $S$. cerevisiae derived ScRad52 was integrated into the genome of the $Y$. lipolytica strains Polf and Polf${ }^{k}$ u70.Additionally, YlRad52wasknockedouttoinvestigatethe functionofendogenousRad52in Y. lipolytica.Homologyarmsofd

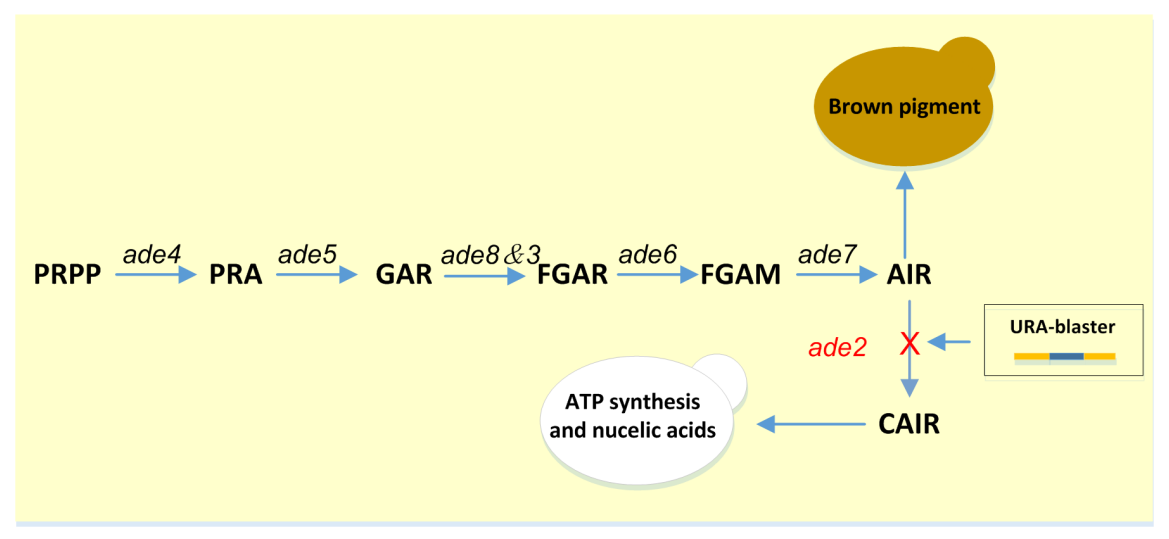




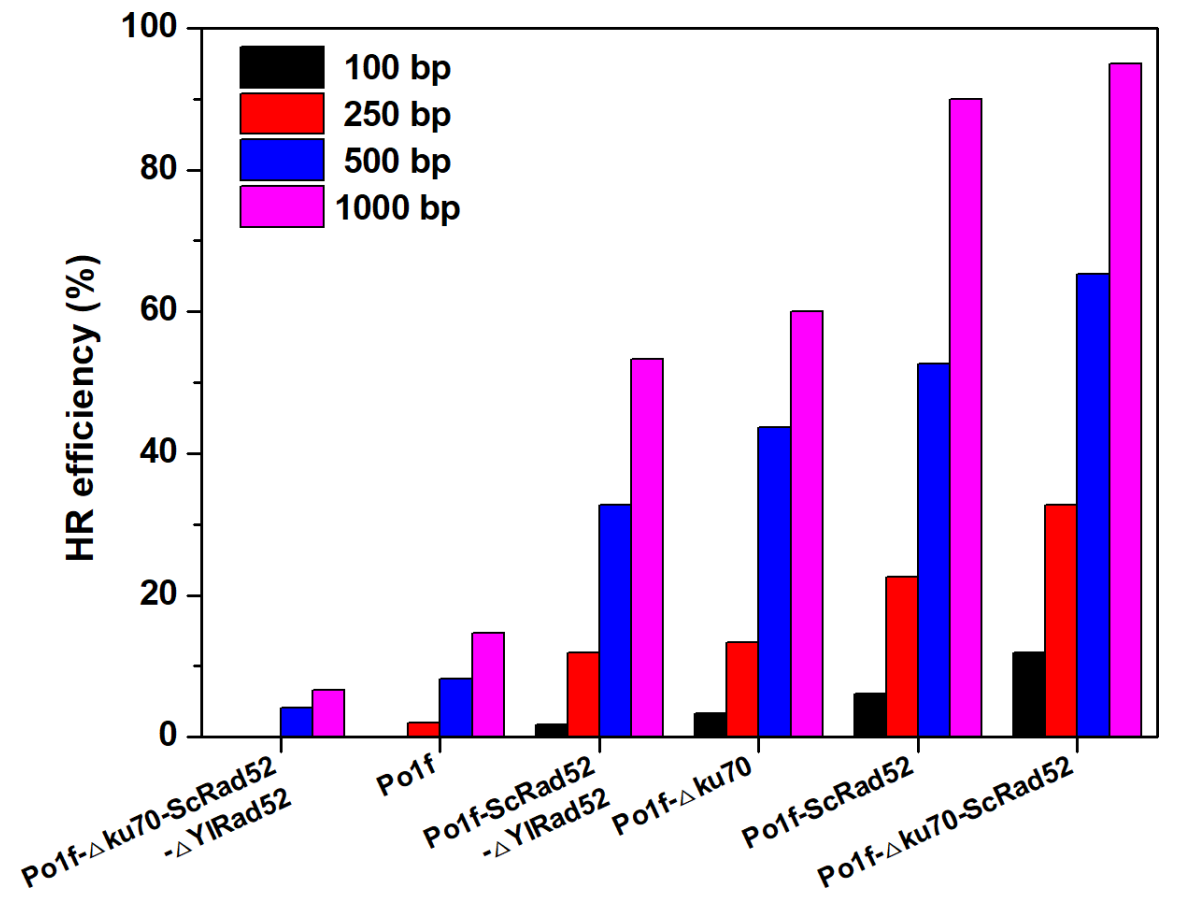

